\section{Mammatumor bei Hyperlipidämie}

Cholesteringranulome der Brust sind seltene gutartige Tumoren, die als radiologische und ultrasonographische Differenzialdiagnose von Bedeutung sein können.

\section{Fallbeschreibung}

Eine 73-jährige Patientin mit einem Mammatumor, der sich mammographisch glatt begrenzt zeigte, wurde uns zuverwiesen. Die Aufnahme der Patientin erfolgte wegen eines Mammatumors links, den die Patientin erstmalig einen Monat vor der stationären Aufnahme selbst tastete.

Der derbe, walnussgroße, verschiebliche Tumor war nicht dolent. Es zeigte sich keine Mamillensekretion und axilläre Lymphknoten ließen sich nicht tasten.

Anamnestisch bestand bei der Patientin eine Hyperlipidämie (Cholesterin $226 \mathrm{mg} / \mathrm{dl}$; Triglyzeride $480 \mathrm{mg} / \mathrm{dl}$ ), die medikamentös mit Bezafibrat behandelt wurde. Zusätzlich bestanden ein diätetisch behandelter Diabetes mellitus, eine Hyperurikämie sowie ein arterieller Hypertonus. In der Vorgeschichte findet sich eine Cholezystektomie. Die Familienanamnese war unauffällig hinsichtlich Mammakarzinomen.

Die durchgeführte Mammographie zeigte einen fast retromamillär gelegenen, dichten, weitgehend glatt begrenzten Tumor (Abb.1). Mammasonographisch fand sich ein $3,4 \times 3,7 \times 2,8 \mathrm{~cm}$ großen, zum Teil unscharf begrenzte Tumor mit fettgleicher Echodensität und inhomogener Echostruktur (Abb. 2). Der Tumor wies ein bilaterales Schallphänomen auf.

Die glatte Begrenzung ließ differenzialdiagnostisch in erster Linie an einen gutartigen Tumor denken, z.B. ein Fibrom, ein Fibroadenom, ein Adenofibrolipom, ein Hämangiom oder eine Zyste. Weniger wahrscheinlich erschien ein maligner Tumor. Differenzialdiagnostisch kamen hier ein muzinöses Mammakarzinom, ein medulläres Karzinom, ein Sarkom oder eine Metastase in Frage.

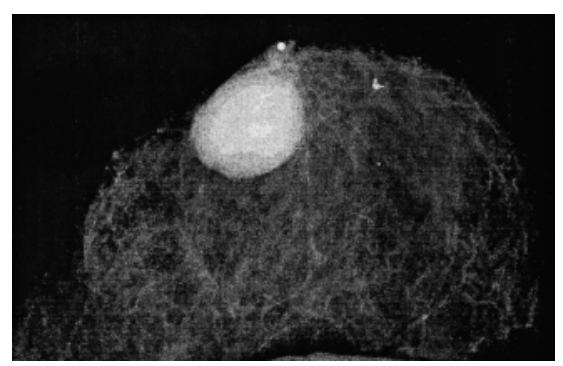

Abb. 1 Mammographie links, kranio-kaudal.

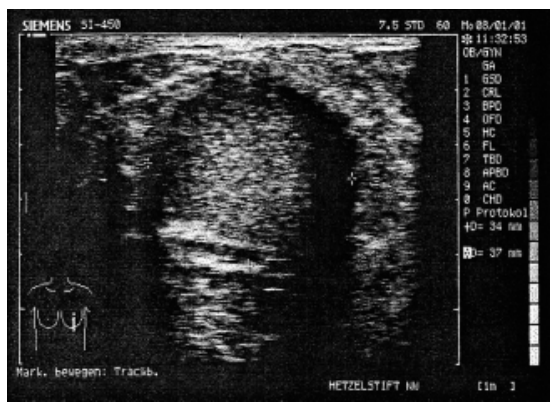

Abb. 2 Mammasonographie links.

Wir führten die Exstirpation des Tumors durch. Histologisch zeigte sich eine $3 \mathrm{~cm}$ große Makrozyste, die ein Cholesteringranulom enthielt. Die Zyste zeigte eine fibrosierte Wandung und, abschnittsweise, typische Epithele mit einzelnen Rundzellinfiltraten. Daneben waren reichlich Cholesterinkristalle, stellen- weise mit Siderinpigmenten, erkennbar. Eine frische Einblutung war nicht nachweisbar. Das Gewebe um die Cholesterolzyste zeigte eine Mastopathia fibrosa et cystica mit kleinherdiger duktaler Hyperplasie (Prechtel II).

\section{Diskussion}

Cholesteringranulome der weiblichen Brust sind sicherlich eine Rarität. Bislang sind nach unserem Kenntnisstand erst drei Fälle berichtet worden, die sich mammographisch und sonographisch vergleichbar darstellten (Smith et al., Br J Radiology 1997; 70: 1178, Reynolds et al., Radiology 1994; 191: 249, Wilhelmus et al., Am J Clin Pathol 1982; 77: 592). Hierbei wurde postuliert, dass Cholesterinkristalle aus ektatischen Milchgängen eine Fremdkörperreaktion auslösen. Ob Hyperlipidämie zu Cholesteringranulomen prädisponiert, ist nach unserem Kenntnisstand nicht gesichert. Häufiger sind Cholesteringranulome in Verbindung mit einem Cholesteatom oder im Bereich des petrösen Apex (Übersicht: Lorenz et al., Laryngorhinootologie 1996; 75: 775). Beschrieben wurde dieser Tumor auch in der Blase (Grunberger, Wien Med Wochenschr 1980; 130: 529) und im Bereich des Foramen mastoideum. Cholesteringranulome sollten als radiologische Differenzialdiagnose bei mammographisch benignen und sonographisch fettisoreflexiblen Strukturen erwogen werden.

P. Deuschle, M. Schmidt-Hieber, Neustadt/W. 